\title{
Strong local moment antiferromagnetic spin fluctuations in V-doped LiFeAs
}

Zhuang Xu ${ }^{1,9}$, Guangyang Dai ${ }^{2,9}$, Yu Li $^{3,4}$, Zhiping Yin ${ }^{1 凶}$, Yan Rong ${ }^{1}$, Long Tian ${ }^{1}$, Panpan Liu ${ }^{1}$, Hui Wang ${ }^{1}$, Lingyi Xing ${ }^{2,5}$, Yuan Wei ${ }^{2}$, Ryoichi Kajimoto $\mathbb{D}^{6}$, Kazuhiko Ikeuchi ${ }^{7}$, D. L. Abernathy ${ }^{8}$, Xiancheng Wang ${ }^{2}$, Changqing Jin ${ }^{2}$, Xingye Lu (D) ${ }^{1}$, Guotai Tan (iD) ${ }^{1 凶}$ and Pengcheng Dai (iD) ${ }^{1,4 凶}$

We use neutron scattering to study Vanadium (hole)-doped LiFe ${ }_{1-x} \mathrm{~V}_{x} A$ s. In the undoped state, LiFeAs exhibits superconductivity at $T_{\mathrm{c}}=18 \mathrm{~K}$ and transverse incommensurate spin excitations similar to electron overdoped iron pnictides. Upon Vanadium doping to form $\mathrm{LiFe}_{0.955} \mathrm{~V}_{0.045}$, the transverse incommensurate spin excitations in LiFeAs transform into longitudinally elongated ones in a similar fashion to that of potassium (hole)-doped $\mathrm{Ba}_{0.7} \mathrm{~K}_{0.3} \mathrm{Fe}_{2} \mathrm{As}_{2}$ but with dramatically enhanced magnetic scattering and elimination of superconductivity. This is different from the suppression of the overall magnetic excitations in hole-doped BaFe $\mathrm{As}_{2}$ and the enhancement of superconductivity near optimal hole doping. These results are consistent with density function theory plus dynamic mean field theory calculations, suggesting that Vanadium doping in LiFeAs may induce an enlarged effective magnetic moment $S_{\text {eff }}$ with a spin crossover ground state arising from the inter-orbital scattering of itinerant electrons.

npj Quantum Materials (2020)5:11; https://doi.org/10.1038/s41535-020-0212-x

\section{INTRODUCTION}

The flexible spin states of $\mathrm{Fe}^{2+}$ ions are interesting due to its extensive distribution, from the lowermost mantle in the earth ${ }^{1}$ to the innermost hemoglobin in our human body ${ }^{2}$ and from the most common ferrite magnet to the most recent iron-based superconductors. ${ }^{3-5}$ Ignoring the orbital angular momentum, each $\mathrm{Fe}^{2+}$ ion with six electrons distributed in five $d$ orbitals has three possible spin states, $S=0,1$, or 2 [Fig. 1a]. In iron-based superconductors, where $\mathrm{Fe}^{2+}$ ion is surrounded by crystal electric field of As atoms, the fivefold degeneracy of the $d$ orbitals is split into a twofold degenerate $e_{g}$ and a threefold degenerate $t_{2 g}$ states nearby the Fermi level [Fig. 1a]. ${ }^{3}$ Although it is widely believed that Hund's rule plays a critical role in determining the electronic configuration of iron, ${ }^{6}$ the $S=2$ spin state has never been observed in metallic iron pnictides. ${ }^{5,7}$

The total moment sum rule within a Heisenberg model for system with spin $S$ requires $\left\langle m^{2}\right\rangle=\left(g \mu_{\mathrm{B}}\right)^{2} S(S+1)$, where $g \approx 2$ is the Landé $g$-factor, when magnetic scattering is integrated over all energy and momentum space within the Brillouin zone (BZ). ${ }^{8}$ Since neutron scattering directly measures the energy $(E)$ and momentum (Q) dependence of the dynamic structure factor $S(\mathbf{Q}, E)$, it is therefore possible to estimate the effective spin-fluctuating moment $S_{\text {eff }}$ by integrating the magnetic spectral weight over all energies and wave vectors within the $B Z$. In previous inelastic neutron scattering on electron-doped $\mathrm{BaFe}_{2} \mathrm{As}_{2}$ compounds, it is established that electron doping via $\mathrm{Ni}$ substitution in $\mathrm{BaFe}_{2}$ ${ }_{-} \mathrm{Ni}_{x} \mathrm{As}_{2}$ reduces slightly the total spin-fluctuating moment, changing from $\left\langle m^{2}\right\rangle \approx 3.5 \mu_{\mathrm{B}}^{2} / \mathrm{Fe}$ at $x=0$ to $\left\langle m^{2}\right\rangle \approx 2.7 \mu_{\mathrm{B}}^{2} / \mathrm{Fe}$ at $x=0.3{ }^{9,10,12}$ These values approximately correspond to $S_{\text {eff }} \approx$ $1 / 2$. $^{9,11}$ For hole-doped $\mathrm{Ba}_{1-x} \mathrm{~K}_{x} \mathrm{Fe}_{2} \mathrm{As}_{2}$, the total spin-fluctuating moment decreases rapidly with increasing $x_{1}{ }^{9}$ and $S_{\text {eff }}$ reduces to 0.14 at $x=1 .{ }^{13}$ For electron-doped $\mathrm{NaFe}_{1-x} \mathrm{Co}_{x} \mathrm{As}^{5}{ }^{5}$ the total spinfluctuating moment also decreases slightly with increasing $x$ but has almost identical value as that of $\mathrm{BaFe}_{2-x} \mathrm{Ni}_{x} \mathrm{As}_{2},{ }^{14}$ thus suggesting universal nature of the $S_{\text {eff }} \approx 1 / 2$ for two different families of iron pnictides. This is different from the superconducting LiFeAs, where the low-energy spin fluctuations coupled to superconductivity are about a factor 4 smaller than that of the Co-underdoped superconducting $\mathrm{BaFe}_{2} \mathrm{As}_{2} \cdot{ }^{15,16}$ The total fluctuating moment of LiFeAs is $\left\langle m^{2}\right\rangle \approx 1.5 \pm 0.3 \mu_{\mathrm{B}}^{2} / \mathrm{Fe}$, more than a factor of 2 smaller than that of $\mathrm{BaFe}_{2} \mathrm{As}_{2} \cdot{ }^{17,18}$ On the other hand, the total spin-fluctuating moment for iron chalcogenide compounds FeSe and FeTe are significantly larger. In the case of FeSe, the total spin-fluctuating moment of $\left\langle\mathrm{m}^{2}\right\rangle \approx 5.2 \mu_{\mathrm{B}}^{2} / \mathrm{Fe}$ arises entirely from dynamic magnetic excitations as the system has no static ordered moment. ${ }^{19}$ For FeTe, the effective moment of Fe may change from $S_{\text {eff }} \approx 1$ at $10 \mathrm{~K}$ to $S_{\text {eff }} \approx 3 / 2$ at $300 \mathrm{~K}$, suggesting entangled local magnetic moments with the itinerant electrons on warming. ${ }^{20}$

The total moment sum rule strictly speaking is only valid for insulating local moment systems, where quantum effect and itinerant electron-induced magnetism are not important. ${ }^{8}$ In ironbased superconductors, itinerant electrons and hole-electron Fermi surface nesting are known to be important for magnetism and superconductivity [Fig. 1b]. ${ }^{21}$ Theoretically, many physical properties of iron-based superconductors can be understood within the orbital-selective Mott phase picture, where electrons in $\mathrm{Fe}^{2+}$ ion with different orbitals can behave differently. ${ }^{22-24}$ In this model, hole-doped compounds are believed to be more correlated and therefore should have large effective local moments. ${ }^{22-24}$ From combined density functional theory (DFT)

\footnotetext{
${ }^{1}$ Center for Advanced Quantum Studies and Department of Physics, Beijing Normal University, 100875 Beijing, China. ${ }^{2}$ Beijing National Laboratory for Condensed Matter Physics, Institute of Physics, Chinese Academy of Sciences, 100190 Beijing, China. ${ }^{3}$ Louisiana Consortium for Neutron Scattering and Department of Physics and Astronomy, Louisiana State University, Baton Rouge, LA 70803, USA. ${ }^{4}$ Department of Physics and Astronomy, Rice University, Houston, TX 77005, USA. ${ }^{5}$ Department of Physics and Astronomy, Louisiana State University, Baton Rouge, LA 70803, USA. ${ }^{6}$ Materials and Life Science Division, J-PARC Center, Japan Atomic Energy Agency, Tokai, Ibaraki 319-1195, Japan. ${ }^{7}$ Neutron Science and Technology Center, Comprehensive Research Organization for Science and Society (CROSS), Tokai, Ibaraki 319-1106, Japan. ${ }^{8}$ Neutron Scattering Division,

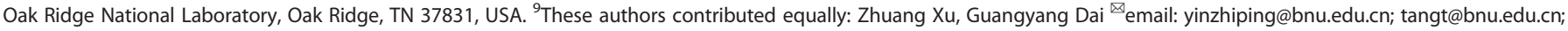
pdai@rice.edu
} 
(a)
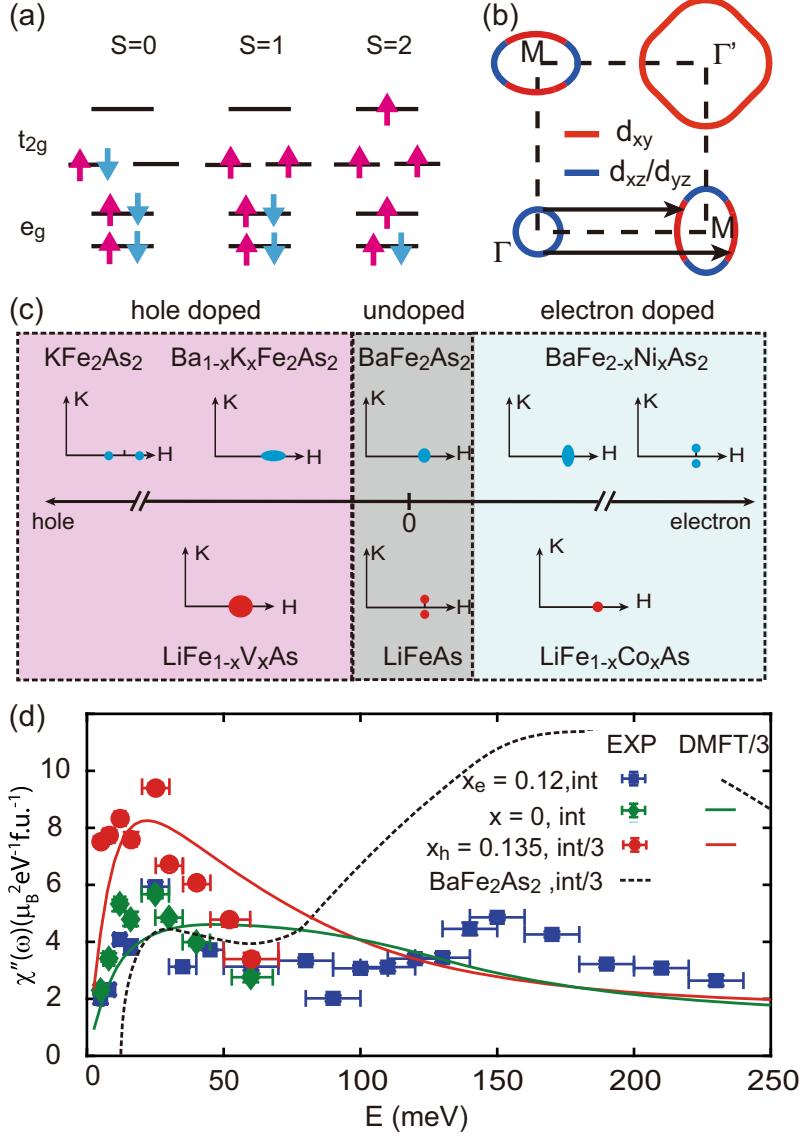

Fig. 1 Summary of neutron-scattering results of hole- and electron-doped LiFeAs. a Possible spin states of $\mathrm{Fe}^{2+}$ in $t_{2 g}$ and $e_{g}$ states within a localized moment picture. b Fermi surfaces of $\mathrm{LiFe}_{0.955} \mathrm{~V}_{0.045} \mathrm{As}$. Red and blue colors represent $d_{x y}$ and $d_{x z / y z}$ orbital characters, respectively. c Summary of the doping dependence of low-energy spin excitations in $\mathrm{BaFe}_{2} \mathrm{As}_{2}$ (Top) and LiFeAs (bottom). The shapes of the magnetic scattering are marked by blue $\left(\mathrm{BaFe}_{2} \mathrm{As}_{2}\right)$ and red (LiFeAs) colored points. It is worth noting that spin excitations in $\mathrm{LiFe}_{0.955} \mathrm{~V}_{0.045} \mathrm{As}$ are considerably diffusive in reciprocal space. d Theoretical and experimental local dynamical spin susceptibility in LiFeAs $(x=0), \mathrm{LiFe}_{0.88} \mathrm{Co}_{0.12} \mathrm{As}\left(x_{e}=0.12\right)$, $\mathrm{LiFe}_{0.955} \mathrm{~V}_{0.045} \mathrm{As}\left(x_{h}=0.135\right)$, and $\mathrm{BaFe}_{2} \mathrm{As}_{2}$.

and dynamic mean field theory (DMFT) calculations, ${ }^{6}$ the total fluctuating moments in the paramagnetic state are nearly invariant around $2.2 \mu_{\mathrm{B}} / \mathrm{Fe}$ in a wide variety of iron pnictide/ chalcogenide families. In X-ray emission spectroscopy (XES) experiments, values of magnetic spin moments in the paramagnetic phase are about $1.3 \mu_{\mathrm{B}} / \mathrm{Fe}$ for iron pnictides, including $\mathrm{BaFe}_{2} \mathrm{As}_{2}$ and $\mathrm{Ba}(\mathrm{Fe}, \mathrm{Co})_{2} \mathrm{As}_{2}$. ${ }^{7}$ These values are considerably smaller than the predictions from DFT+DMFT calculation but larger than those from neutron-scattering experiments, ${ }^{9,10,12}$ possibly due to the fast time scale of the XES measurements. ${ }^{7}$ Therefore, to fully understand the key ingredients of magnetism in iron-based superconductors, it is important to study the evolution of $S_{\text {eff }}$ in different families of hole-doped iron-based superconductors in a controlled and systematic way.

While it is easy to dope holes in $\mathrm{BaFe}_{2} \mathrm{As}_{2}$ family of iron pnictides by substituting $\mathrm{Ba}^{2+}$ cation with $\mathrm{K}^{1+}$, replacing $\mathrm{Fe}$ with $\mathrm{Cr} / \mathrm{Mn}$ introduces impurities and/or disorders instead of holes. ${ }^{25-27}$ For NaFeAs family, systematic neutron scattering and angleresolved photoemission spectroscopy (ARPES) experiments reveal that replacing $\mathrm{Fe}$ with $\mathrm{Cu}$ dopes holes into the system and induces large ordered magnetic moment for $x>0.1$ in $\mathrm{NaFe}_{1-x} \mathrm{Cu}_{x} \mathrm{As}^{28,29}$ However, there are no inelastic neutron-scattering experiments to determine the total fluctuating moment for this family of compounds. In the case of LiFeAs family, ARPES experiments indicate that doping $\mathrm{V}$ into Fe sites actually introduces hole carriers with a rate of 0.3 hole per $\mathrm{V}$ dopant and selectively enlarges the inner hole Fermi surface [Fig. 1b], ${ }^{30}$ even though the doping-induced impurity and disorder effects might still play a role in the transport and susceptibility measurements with higher $\mathrm{V}$ doping ratio. Although hole doping via $\mathrm{K}$ substitution in $\mathrm{Ba}_{1}$ ${ }_{-x} \mathrm{~K}_{x} \mathrm{Fe}_{2} \mathrm{As}_{2}$ induces superconductivity, $<2 \% \mathrm{~V}$ doping in $\mathrm{LiFe}_{1}$ ${ }_{-x} \mathrm{~V}_{x}$ As quickly suppresses the superconductivity in pure LiFeAs, even with a perfect nesting condition established between the inner hole and electron Fermi pockets at $x=0.084$ [see arrows in Fig. 1b]. ${ }^{30}$ The rapid suppression of superconductivity in $\mathrm{LiFe}_{1}$ ${ }_{-x} \mathrm{~V}_{x}$ As has been suggested as due to magnetic impurity effect of $\mathrm{V}$ dopant, but there are no neutron-scattering experiments to establish the effect of $\mathrm{V}$ doping to spin excitations of pure LiFeAs . ${ }^{15-17}$

\section{RESULTS}

We report the magnetic excitations in hole-doped nonsuperconducting $\mathrm{LiFe}_{0.955} \mathrm{~V}_{0.045} \mathrm{As}$, which has nearly nested hole-electron Fermi surfaces with $d_{x z / y z}$ and $d_{x y}$ orbital characters, respectively, and exhibits non-Fermi-liquid behavior. ${ }^{30}$ We observe enhanced commensurate magnetic fluctuations at antiferromagnetic (AF) wave vectors, suggesting that the non-Fermi-liquid behavior is intimately associated with magnetic fluctuations. The broadness and diffuseness of the spin excitations are also distinct from the sharp commensurate spin fluctuations in electron-doped $\mathrm{LiFe}_{0.88^{-}}$ $\mathrm{CO}_{0.12} \mathrm{As}^{18}$ probably reflecting the local nature of quantum spin fluctuations. ${ }^{31}$ More importantly, we find that the intensities of spin fluctuations are strongly enhanced in the energy range of our measurements with a larger $S_{\text {eff, }}$ in sharp contrast to the reduced effective moment in hole-doped $\mathrm{Ba}_{1-x} \mathrm{~K}_{x} \mathrm{Fe}_{2} \mathrm{As}_{2}{ }^{9,13}$ Considering the different superconducting behavior in $\mathrm{Ba}_{1-x} \mathrm{~K}_{x} \mathrm{Fe}_{2} \mathrm{As}_{2}$ and $\mathrm{LiFe}_{1-x} \mathrm{~V}_{x} \mathrm{As}$, we argue that the inter-orbital scattering processes between $d_{x z / y z}$ and $d_{x y}$ orbitals increase the fluctuating moment but are detrimental to superconductivity. ${ }^{32}$ These results are consistent with the notion that intra-orbital scattering processes between hole and electron pockets, particularly those involving $d_{y z}-d_{y z}$ orbital characters, are good for superconductivity in different classes of iron-based superconductors. ${ }^{33-37}$

We first plot in Fig. 2 the two-dimensional images of spin excitations in $\mathrm{LiFe}_{0.955} \mathrm{~V}_{0.045} \mathrm{As}$ at different energies and their comparison with the DFT+DMFT calculations for $10 \%$ hole doping (about $\mathrm{LiFe}_{0.967} \mathrm{~V}_{0.033} \mathrm{As}$ ). At energy transfer $E=3 \pm 1 \mathrm{meV}$, spin excitations occur at commensurate $Q_{A F}=(1,0)$ and $(0,1)$ positions [Figs. 1c and 2a], different from the transverse incommensurate spin fluctuations in LiFeAs. ${ }^{15-17}$ Instead, spin excitations display a small elongation along the longitudinal direction, mimicking the low-energy spin excitations of hole-doped $\mathrm{Ba}_{1-x} \mathrm{~K}_{x} \mathrm{Fe}_{2} \mathrm{As}_{2}{ }^{38}$ As the energy transfer increases from $E=3$ to $E=18 \mathrm{meV}$, the elongation of the elliptical spin fluctuations changes from the longitudinal to transverse direction, similar to that in $\mathrm{Ba}_{0.67} \mathrm{~K}_{0.33} \mathrm{Fe}_{2} \mathrm{As}_{2}$, reflecting the hole-electron Fermi surface nesting contribution from itinerant electrons. ${ }^{32,38}$ The wave vector-dependent spectra at the corresponding energies are confirmed by the DFT+DMFT calculations in the $10 \%$ hole-doped LiFeAs [Fig. 2e-h]. These results are significantly different from $\mathrm{Mn}$-substituted $\mathrm{BaFe}_{2} \mathrm{As}_{2}$, where $\mathrm{Mn}$ doping induces diffusive magnetic scattering at the checkerboard wave vector $(1,1) .{ }^{27}$ The broad and diffusive magnetic scattering at $(1,0) /(0,1)$ may be attributed to the shortrange spin fluctuations from localized moment and/or not perfect nesting condition between hole and electron Fermi surfaces.

To compare the magnitude of spin fluctuations in LiFeAs, $\mathrm{LiFe}_{0.88} \mathrm{Co}_{0.12} \mathrm{As}$, and $\mathrm{LiFe}_{0.955} \mathrm{~V}_{0.045} \mathrm{As}$, we calculate in Fig. $1 \mathrm{~d}$ the energy dependence of the local dynamic spin susceptibility $X^{\prime \prime}(E)$ for these materials obtained by integrating wave vector- 

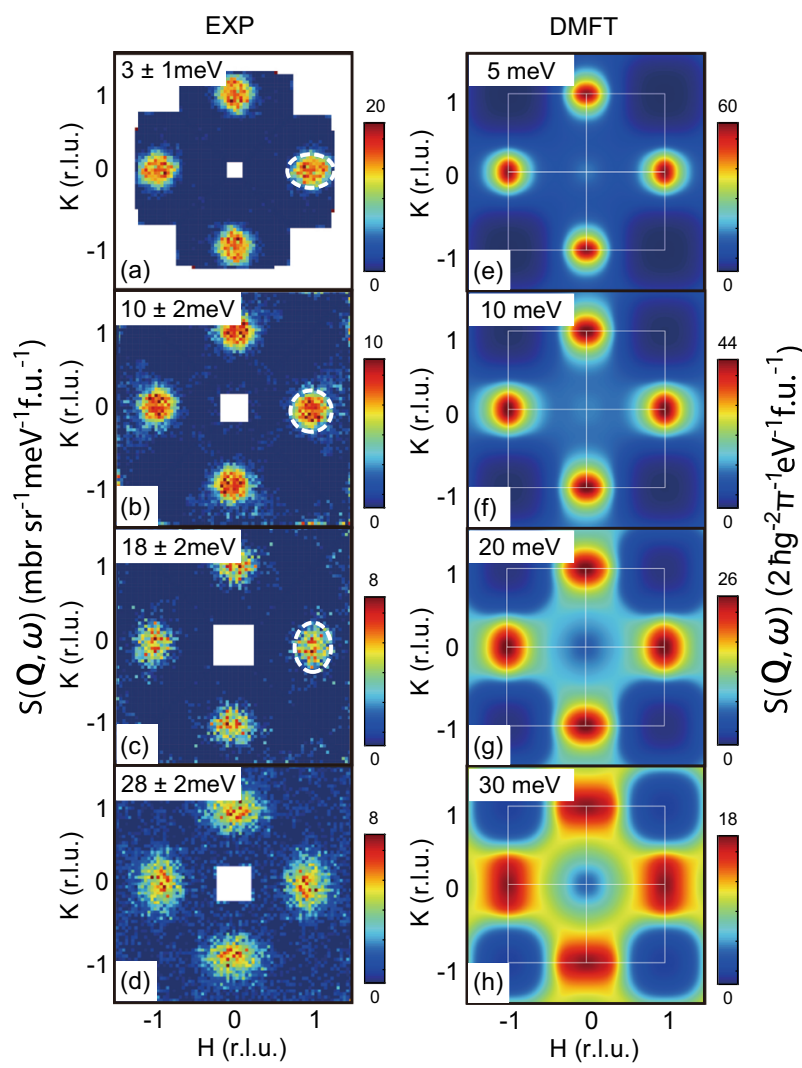

Fig. 2 Comparison of energy dependence of the magnetic scattering of $\mathrm{LiFe}_{0.955} \mathrm{~V}_{0.045} \mathrm{As}$ with that of DFT+DMFT calculations. a-d 2D images of measured dynamic spin susceptibility of $\mathrm{LiFe}_{0.955} \mathrm{~V}_{0.045} \mathrm{As}$ in the $[H, K]$ plane at $E=3 \pm 1,10 \pm 2,18 \pm 2$, and $28 \pm 2 \mathrm{meV}$, respectively. The elongation of spin excitations along $[H, 0,0]$ can be observed in both experimental (dashed circle) and theoretical (green and red area) results. The data were collected at $T=5 \mathrm{~K}$ and folded to improve statistics with radially symmetric backgrounds subtracted. e-h The corresponding results from DFT +DMFT calculations. The color bars indicate scattering intensity in absolute units from experiments and theory.

dependent dynamic spin susceptibility $X^{\prime \prime}(\mathbf{Q}, E)$ over the entire BZ. ${ }^{5}$ Compared with those of LiFeAs and $\mathrm{LiFe}_{0.88} \mathrm{CO}_{0.12} \mathrm{As}^{18}{ }^{18} X^{\prime \prime}(E)$ in $\mathrm{LiFe}_{0.955} \mathrm{~V}_{0.045}$ As is clearly enhanced about $<80 \mathrm{meV}$, although we do not have data $>80 \mathrm{meV}$. Such an enhancement is also captured in the DFT+DMFT calculations as shown in red and blue solid lines of Fig. $1 \mathrm{~d}$. The local dynamic spin susceptibility of $\mathrm{LiFe}_{0.955} \mathrm{~V}_{0.045} \mathrm{As}$ in the measured energy range is somewhat larger than that of $\mathrm{BaFe}_{2} \mathrm{As}_{2} \cdot{ }^{39,40}$ For comparison, magnetic scattering of LiFeAs is considerably smaller than that of the Co-doped superconducting $\mathrm{Ba}\left(\mathrm{Fe}_{0.92} \mathrm{CO}_{0.08}\right)_{2} \mathrm{As}_{2}$. ${ }^{15,16}$

The differences between LiFeAs, $\mathrm{LiFe}_{0.88} \mathrm{Co}_{0.12} \mathrm{As}$, and $\mathrm{LiFe}_{0.955^{-}}$ $\mathrm{V}_{0.045}$ As can be further confirmed in Fig. $3 a$ in which we plot constant-energy cuts of spin excitations along the transverse and longitudinal directions. In all cases, we obtain the magnitude of magnetic scattering by normalizing it to phonon intensities, ${ }^{14}$ making it possible to compare magnetic scattering of different materials measured on different instruments. It is clear that the magnetic scattering in $\mathrm{LiFe}_{0.955} \mathrm{~V}_{0.045} \mathrm{As}$ is both wider and higher than those in LiFeAs and $\mathrm{LiFe}_{0.88} \mathrm{Co}_{0.12} \mathrm{As}$ at $E=5 \mathrm{meV}$, resulting in a large increase in $X^{\prime \prime}(E)$ [Fig. 1d]. This cannot be explained by the extra Vanadium magnetic impurities since the $3 d^{3}$ electronic configuration of $\mathrm{V}^{2+}$ ions only has $S=3 / 2$, which is smaller than the expected $S=2$ for $3 d^{6}$ of $\mathrm{Fe}^{2+}$ in iron-based superconductors. Therefore, the enhanced magnetic scattering has to be associated

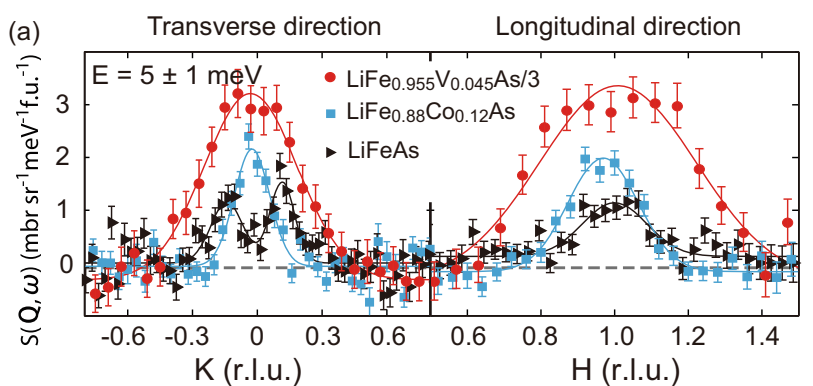

(b)

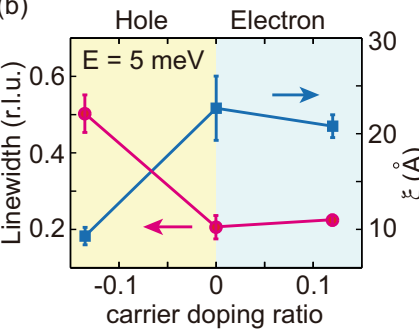

(c)

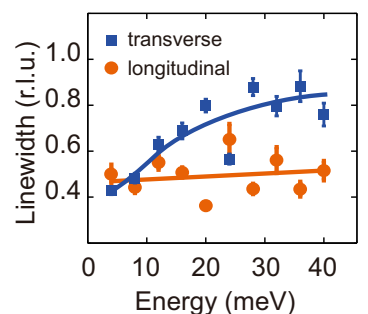

Fig. 3 Comparison of normalized magnetic scattering of holeand electron-doped LiFeAs in absolute units. a Constant-energy cuts of spin excitations along the transverse $[1, K]$ (left) and longitudinal $[H, 0]$ (right) directions for $\mathrm{LiFeAs}, \mathrm{LiFe}_{0.88} \mathrm{Co}_{0.12} \mathrm{As}$, and $\mathrm{LiFe}_{0.955} \mathrm{~V}_{0.045}$ As at $E=5 \pm 1 \mathrm{meV}$, respectively. The solid lines are corresponding single- or two-Gaussian fits. The intensity is normalized by relative phonon intensity to account for spectrometer differences. ${ }^{14} \mathbf{b}$ Line width and correlation length of the low energy spin fluctuations along the longitudinal $[H, 0]$ direction in LiFeAs and hole/electron-doped compounds. The doping ratio is a theoretical value assuming that each V dopant contributes three holes. c Energy dependence of the line widths of the low energy spin fluctuations in $\mathrm{LiFe}_{0.955} \mathrm{~V}_{0.045} \mathrm{As}$ along the transverse and longitudinal directions, respectively.

with the introduction of hole carriers, which effectively modifies the spin configuration of $\mathrm{Fe}^{2+}$ ions.

In Fig. 3b, we show the line width of spin excitations and the corresponding dynamic spin-spin correlation length for LiFeAs, $\mathrm{LiFe}_{0.88} \mathrm{Co}_{0.12} \mathrm{As}$, and $\mathrm{LiFe}_{0.955} \mathrm{~V}_{0.045}$ As. The sudden drop of spin correlation length in $\mathrm{LiFe}_{0.955} \mathrm{~V}_{0.045} \mathrm{As}$ suggests that short-range spin correlations of local moments may become important. Figure $3 \mathrm{c}$ shows the line widths of spin excitations along both the transverse and longitudinal directions at different energies in $\mathrm{LiFe}_{0.955^{-}}$ $\mathrm{V}_{0.045} \mathrm{As}$. The crossing around $10 \mathrm{meV}$ is consistent with the isotropic spin excitations in Fig. 2b. Therefore, $\mathrm{LiFe}_{1-x} \mathrm{~V}_{x} \mathrm{As}$ is distinct from $\mathrm{Cr} / \mathrm{Mn}$-doped $\mathrm{BaFe}_{2} \mathrm{As}_{2}$ in which magnetic impurities induce Néel-type diffusive AF spin fluctuations at low concentrations. $^{25-27}$

Figure $4 \mathrm{a}, \mathrm{b}$ compare the DFT+DMFT calculated wave vector and energy dependence of $\chi^{\prime \prime}(\mathbf{Q}, E)$ for LiFeAs and $\mathrm{LiFe}_{0.967} \mathrm{~V}_{0.033} \mathrm{As}$, respectively. Consistent with Fig. $1 \mathrm{~d}$, we find that $\mathrm{LiFe}_{0.967} \mathrm{~V}_{0.033} \mathrm{As}$ has larger magnetic spectral weight, especially at low energies. In Fig. 4c, we show the estimated effective spin $S_{\text {eff }}$ for different ironbased superconductors. It is clear that all values are distributed between $S=0$ and $S=1$ with most of them around $S_{\text {eff }}=1 / 2$. The discrepancy of hole-doping dependence of the magnetic excitations between $\mathrm{BaFe}_{2} \mathrm{As}_{2}$ and LiFeAs families may arise mostly from the fact that we have only integrated the energy range of $X^{\prime \prime}(E)$ from 0 to about $60 \mathrm{meV}$ where we have solid data for V-doped and pure LiFeAs. Although these numbers cannot be compared with absolute values of $\mathrm{S}_{\text {eff }}$ determined for $\mathrm{BaFe}_{2} \mathrm{As}_{2}$ and FeSe, the trend of increasing total magnetic susceptibility with hole doping in V-doped LiFeAs is unmistakable. Therefore, it is interesting to understand the microscopic origin of magnetic excitations in V-doped LiFeAs. 
(a)

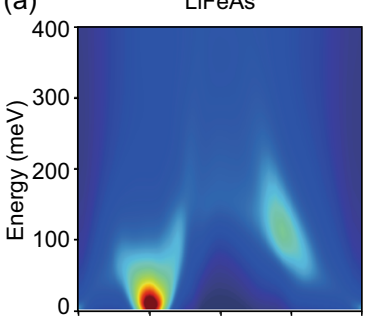

$(0,0) \quad(1,0) \quad(1,1)(0.5,0.5)(0,0)(0,0) \quad(1,0) \quad(1,1)(0.5,0.5)(0,0)$

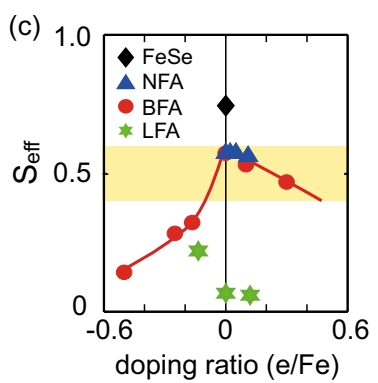

(d)

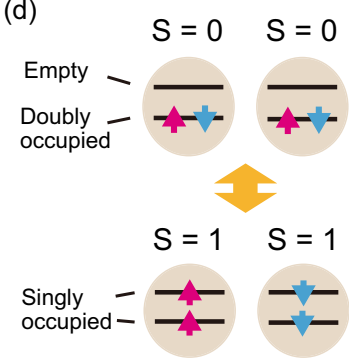

Fig. 4 Comparison of the DFT+DMFT-calculated magnetic scattering of LiFeAs and $\mathrm{LiFe}_{0.967} \mathbf{V}_{\mathbf{0 . 0 3 3}}$ As. $\mathbf{a}$, b The calculated $S(\mathbf{Q}, E)$, obtained by using computed dynamical spin susceptibility $X^{\prime \prime}(\mathbf{Q}, E)$ and $\chi^{\prime \prime}(\mathbf{Q}, E)=g^{2} \mu_{\mathrm{B}}^{2} \frac{\pi}{\hbar}\left(1-\exp \left(-\hbar \omega / k_{\mathrm{B}} T\right)\right) S(\mathbf{Q}, E)$ of LiFeAs and $10 \%$ holedoped $\mathrm{LiFe}_{0.967} \mathrm{~V}_{0.033}$ As by DFT+DMFT. ${ }^{5}$ They are plotted in the same color scale for direct comparison. The factor 2 in the units comes from a factor of 2 in the formula that we did not multiply when we wrote the code to compute $x^{\prime \prime}$. The color bar indicates scattering intensity in absolute units from the DFT+DMFT calculation. c The effective spin $S_{\text {eff }}$ in various iron pnictide/chalcogenides superconductors obtained from neutron-scattering experiments. ${ }^{12-14,18,19,54}$ NFA, BFA, and LFA represent NaFeAs, $\mathrm{BaFe}_{2} \mathrm{As}_{2}$, and LiFeAs families, respectively. The energy integration range of $\chi^{\prime \prime}(E)$ for LiFeAs family of materials is from 0 to about $60 \mathrm{meV}$ and thus does not represent the total local dynamic susceptibility. d Schematics of dynamic mixing of spin states with $S=0$ and 1 .

\section{DISCUSSION}

Theoretically, the reduction of effective spins in iron pnictides/ chalcogenides can be understood in either an itinerant or a localized moment picture. The former considers $\mathrm{Fe}^{2+}$ ions embedded in a Fermi sea of conduction electrons, ${ }^{41,42}$ and the lower spin states can be achieved through a multiple-stage Kondo screening process, ${ }^{31,43}$ where the system is in a quantum critical incoherent metallic state down to $0 \mathrm{~K}$. In the local moment scenario, due to the sensitivity of spin states of $\mathrm{Fe}^{2+}$ to the ionic radius, the effective magnetic moment in iron-based material can be tuned by controlling the strength of crystal electric field in the local FeAs 4 tetrahedral structure. ${ }^{44,45}$ This picture can successfully explain some phenomena such as temperature-dependent magnetic moments ${ }^{46,47}$ and coherent-incoherent crossover. ${ }^{31,48-51}$ However, it is still difficult to understand the large variety of the fluctuated moments in iron-based superconductor by assuming a reduced spin state with $S=1$ or 0 . To achieve an intermediate crossover between spin state $S=1$ and $S=0$, a dynamic "spin mixing" process was proposed ${ }^{44,45}$ as schematically shown in Fig. 4d. When two $\mathrm{Fe}^{2+}$ ions exchange their electrons, their spin states would effectively change from $S=0$ to $S=1$ and vice versa. This results in a continuous evolution of the effective spin state with $0<S_{\text {eff }}<1$. In this scenario, the probability of each $S=0$ or 1 state is closely related to both the local structure and conduction electrons. ${ }^{44}$ We note that this spin crossover scenario is a localized model and may not completely reflect the itinerant nature of ironbased superconductors. Further theoretical and experimental evidences are needed to clarify the situation.
In insulating copper oxides, local moments are locked into the $S=1 / 2$ state by the elimination of doubly occupied states with a large charge gap. ${ }^{52}$ Doping charge carriers introduce doubly occupied states and lead to the destruction of local moments, resulting in the suppression of the neutron-scattering spectral weight in the energy range of typical neutron-scattering experiments. ${ }^{8}$ Similarly, in iron pnictides, the effective moment on each $\mathrm{Fe}^{2+}$ ion is also affected by the population of doubly occupied states or spin singlets due to Kondo screening. However, this cannot be simply attributed to the introduction of charge carriers since the doping dependence of $S_{\text {eff }}$ in $\mathrm{Ba}_{1-x} \mathrm{~K}_{x} \mathrm{Fe}_{2} \mathrm{As}_{2}$ and $\mathrm{LiFe}_{1}$ ${ }_{-x} \mathrm{~V}_{x} \mathrm{As}$ is rather different. Such a difference may be rooted in the different sites of the dopants. Since Vanadium is directly introduced into the FeAs layer while $\mathrm{K}^{+}$is not, the Fe-As bond length is affected by $V$ doping ${ }^{30}$ and a high spin state is favored in V-doped LiFeAs. With increasing V- doping, the nesting condition improves between the inner hole Fermi surface with $d_{x z / y z}$ orbital character and the inner electron pocket mainly derived from $d_{x y}$ orbital character. ${ }^{30}$ Therefore, the enhanced inter-orbital scattering between $d_{x z / y z}$ and $d_{x y}$ orbitals, which suppresses superconductivity, ${ }^{32}$ favors the "spin mixing" process [Fig. 4d] and enhances fluctuated moments. This is different from the intraorbital $d_{y z}-d_{y z}$ hole-electron Fermi surface nesting scattering, which favors superconductivity and induces a neutron spin resonance only at the $\mathrm{AF}$ wave vector in detwinned $\mathrm{FeSe}^{33}$ and underdoped iron pnictides. ${ }^{37,53}$

To conclude, we performed time-of-flight neutron-scattering measurements on $\mathrm{LiFe}_{0.955} \mathrm{~V}_{0.045} \mathrm{As}$ and did DFT+DMFT calculation on the corresponding $10 \%$ hole-doped LiFeAs system. We found that the low-energy spin fluctuations are influenced by itinerant electrons following the same doping dependence of spin excitations in $\mathrm{BaFe}_{2} \mathrm{As}_{2}$. More importantly, both the experimental and theoretical results suggest that magnetic excitations are dramatically enhanced by doping holes with Vanadium. We discuss the possible origin of such enhancement and argue that the inter-orbital scattering between $d_{x z / y z}$ and $d_{x y}$ orbitals are bad for superconductivity and may dynamically mix the spin states of $\mathrm{Fe}^{2+}$ ions.

\section{METHODS}

Sample preparation

We grew single crystals of $\mathrm{LiFe}_{0.955} \mathrm{~V}_{0.045} \mathrm{As}$ by self-flux method. ${ }^{55}$ In all, $7.2 \mathrm{~g}$ of single crystals were used in our time-of-flight neutron-scattering experiments, of which $1.8 \mathrm{~g}$ were synthesized by using isotope ${ }^{7} \mathrm{Li}$ to reduce neutron absorption. Samples were wrapped by Aluminum foil with Hydrogen-free glue in the glove box filled with Argon gas since the samples are air and humidity sensitive. All samples were co-aligned and glued on five $40 \times 40 \mathrm{~mm}^{2}$ Aluminum plates as shown in Fig. S1b. The [1, 0 , 0] direction is along the horizontal direction and $[1,1,0]$ along the diagonal [Fig. S1a].

\section{Neutron-scattering experiments}

Our inelastic neutron-scattering measurements on $\mathrm{LiFe}_{0.955} \mathrm{~V}_{0.045} \mathrm{As}$ were carried out on the time-of-flight 4D-Space Access Neutron Spectrometer (4SEASONS) at Materials and Life Science Experimental Facility (MLF), Japan Proton Accelerator Research Complex (J-PARC). ${ }^{56}$ We define the momentum transfer $Q$ in three-dimensional reciprocal space in $\AA^{-1}$ as $\mathbf{Q}=\mathrm{Ha}^{*}+$ $K \mathbf{b}^{*}+L \mathbf{C}^{*}$, where $H, K$, and $L$ are Miller indices and $\mathbf{a}^{*}=\hat{\mathbf{a}} 2 \pi / a, \mathbf{b}^{*}=\hat{\mathbf{b}} 2 \pi / b$, and $\mathbf{c}^{*}=\hat{\mathbf{c}} 2 \pi / c$ with $a=b \approx 5.316 \AA$, and $c=6.315 \AA .{ }^{17,18}$ Samples are coaligned in the $[H, O, L]$ scattering plane with mosaic $<5^{\circ}$ and incident beam $\left(E_{i}=16,39,75\right.$, and $\left.200 \mathrm{meV}\right)$ parallel to the $c$-axis of the crystals. In principle, absolute magnetic neutron-scattering intensity from $\mathrm{LiFe}_{0.955^{-}}$ $\mathrm{V}_{0.045}$ As can be normalized by comparing the scattering with a Vanadium standard. ${ }^{5}$ However, $\mathrm{LiFe}_{0.955} \mathrm{~V}_{0.045} \mathrm{As}$ has considerable unknown amount of Vanadium-containing flux from the growth, rendering this method unreliable. To obtain the absolute intensity, we therefore use phonon normalization method as discussed below. 
Background subtraction and data analysis

The background subtraction processes are shown in Fig. S2. ${ }^{14}$ The raw magnetic scattering appears in a fourfold symmetry at wave vectors $[1,0]$, $[0,1],[-1,0]$, and $[0,-1]$ [Fig. S2a] due to the tetragonal symmetry of the lattice structure. We symmetrized the scattering spectra in order to enhance the magnetic signal and then remove the background [Fig. S2b]. Background was obtained by masking the signal within the white squares [Fig. S2c] and assumed to be radially symmetric. A polynomial function of Q was used to fit the background intensity [Fig. S2e] and then was subtracted from the raw data. The results were shown in Fig. S2d. Figure S2f, $g$ are cuts along the $[H, 0]$ and $[1, K]$ directions, respectively. We note that there are still residual background intensities, which likely come from phonon scattering and affect the calculation of local dynamical spin susceptibility. Different treatments to these intensities might result in the differences of the final estimated local dynamical spin susceptibility. Therefore, we recalculate the local dynamical spin susceptibility of pure $\mathrm{LiFeAs}$ and $\mathrm{LiFe}_{0.88} \mathrm{Co}_{0.12} \mathrm{As}$ from the previous data with the same method we used for V-doped LiFeAs. In this way, we can have a better comparison between different compounds. The recalculated local dynamical spin susceptibilities are shown in Fig. $1 \mathrm{~d}$.

\section{Absolute neutron-scattering intensity normalization}

We performed our inelastic neutron scattering on LiFeAs and $\mathrm{LiFe}_{0.88^{-}}$ $\mathrm{Co}_{0.12} \mathrm{As}$ at the wide Angular-Range Chopper Spectrometer and Cold Neutron Chopper Spectrometer at Spallation Neutron Source, Oak Ridge National Laboratory and on LiFe $0.955 \mathrm{~V}_{0.045}$ As at 4 SEASONS, MLF, J-PARC. The neutron-scattering intensity of the LiFeAs and $\mathrm{LiFe}_{0.88} \mathrm{Co}_{0.12} \mathrm{As}$ have already been normalized by standard Vanadium sample. The neutronscattering intensity of $\mathrm{LiFe}_{0.955} \mathrm{~V}_{0.045} \mathrm{As}$ is normalized by phonon intensities since a standard Vanadium is unavailable during the experiment. As discussed in the main text, $\mathrm{V}$ normalization is actually less reliable in V-doped LeFeAs because of the unknown amount of V-containing flux in the sample. Therefore, phonon normalization using the same phonon for LiFeAs, which we re-analyzed from using previous data, and V-doped LiFeAs should be more reliable than the standard Vanadium scan when comparing the results from different spectrometers. ${ }^{14}$

We identified two phonons, one is around $(1,1)$ and the other originates from $(2,0)$. The incident neutron energy is almost the same, about $39 \mathrm{meV}$ for $\mathrm{LiFe}_{0.955} \mathrm{~V}_{0.045} \mathrm{As}$ and $35 \mathrm{meV}$ for LiFeAs and $\mathrm{LiFe}{ }_{0.88} \mathrm{Co}_{0.12}$ As. The onedimensional cuts of these phonons are shown in Fig. S3, and the integrated intensity of each phonon can then be calculated. By comparing the phonon intensities of different materials, we estimated the scale factor, which is defined as the ratio of phonon intensity between LiFeAs/ $\mathrm{LiFe}_{0.88} \mathrm{Co}_{0.12} \mathrm{As}$ and $\mathrm{LiFe}_{0.955} \mathrm{~V}_{0.045} \mathrm{As}$. Since the intensity of LiFeAs and $\mathrm{LiFe}_{0.88} \mathrm{CO}_{0.12} \mathrm{As}$ has been normalized already by the standard Vanadium scan, we could calculate the absolute value of the intensities in $\mathrm{LiFe}_{0.955} \mathrm{~V}_{0.045} \mathrm{As}$. The data of LiFeAs and $\mathrm{LiFe}_{0.88} \mathrm{CO}_{0.12} \mathrm{As}$ can be used as a cross-check and the absolute value we obtained for $\mathrm{LiFe}_{0.955} \mathrm{~V}_{0.045} \mathrm{As}$ is consistent with these two references.

Figures S3a, b show the acoustic phonon near $(1,1)$ at 4.5 and $5.5 \mathrm{meV}$ [Fig. S3e], and Figs. S3C, d display another phonon around (2,0) [Fig. S3f] at 12.5 and $13.5 \mathrm{meV}$. In Fig. S3g, we plot the estimated factor from Fig. S3a-d. An average scale factor about 200 is obtained.

Alternatively, the incoherent nuclear scattering can be used as another reference to normalize the data. In Fig. S4, we show the incoherent peak for $\mathrm{LiFe}_{0.955} \mathrm{~V}_{0.045} \mathrm{As}$, LiFeAs, and $\mathrm{LiFe}_{0.88} \mathrm{Co}_{0.12} \mathrm{As}$. The incident energy $E_{i}$ is 39 and $75 \mathrm{meV}$ for $\mathrm{LiFe}_{0.955} \mathrm{~V}_{0.045} \mathrm{As}$ and 35 and $80 \mathrm{meV}$ for LiFeAs and $\mathrm{LiFe}_{0.88} \mathrm{Co}_{0.12} \mathrm{As}$. The estimated scale factor is about 70 , significantly smaller than the value from phonon normalization. We note here that the residual flux trapped in the single crystal samples is an issue that may affect the estimation of the absolute value. This has been observed in our previous paper on Co-doped NaFeAs in which a difference of $30 \%$ was observed. ${ }^{14}$ This situation would be even more serious since the $\mathrm{LiFe}_{0.955} \mathrm{~V}_{0.045} \mathrm{As}$ actually was grown out of a nominal composition of $\mathrm{LiFe}_{0.9} \mathrm{~V}_{0.1} \mathrm{As}$. In particular, the neutron incoherent scattering cross-section of $V$ is huge compared with that of $\mathrm{Fe}, \mathrm{Li}$, and As. The trapped flux with rich $\mathrm{V}$ having a large neutron incoherent cross-section would lead to a serious underestimation of the scale factor. Therefore, we argue that the phonon normalization is more accurate than the normalization by incoherent peaks.

More importantly, we emphasize that, even with this smaller scale ratio, we can still observe the enhancement of magnetic scattering in $\mathrm{LiFe}_{0.955^{-}}$ $\mathrm{V}_{0.045} \mathrm{As}$ [Fig. S5], demonstrating the robustness of our conclusion that the low-energy magnetic excitations in V-doped LiFeAs is greatly enhanced. In the main text, we used the scale factor 200 to normalize all the data. In
Fig. S5, we display the local dynamical spin susceptibility of $\mathrm{LiFe}_{0.955} \mathrm{~V}_{0.045} \mathrm{As}$ from both normalization methods, as well as others for comparison.

\section{Transport results}

Figure S6 shows the magnetic susceptibility and electric resistivity in a series of V-doped LiFeAs. The effective magnetic moments were obtained from the fits with a Curie-Weiss form $\frac{1}{x-x_{0}}=(T-\theta) / C$, in which $\theta$ is the Curie temperature and $X_{0}$ is the temperature-independent constant. It is clear that the effective magnetic moment increases with the concentration of Vanadium, as shown in inset of Fig. S6a. Figure S6b shows that the temperature dependence of resistivity of $\mathrm{LiFe}_{1-x} \mathrm{~V}_{x} \mathrm{As}$ varies from $x=0$ to $x=0.15$, showing the evolution from metallic to insulating behavior. A non-Fermi liquid region was identified with $x$ between 0.05 and 0.15 , suggesting enhanced electronic correlation [Fig. S6b inset]. We note that although the susceptibility and resistivity probe the energy scales different from neutron scattering, the increase of moments and resistivity is qualitatively consistent with the enhancement of low-energy magnetic excitations.

\section{DFT+DMFT calculations}

We use DFT+DMFT ${ }^{57}$ to compute the electronic structure and spin dynamics of V-doped LiFeAs in the paramagnetic state. The DFT part is based on the full-potential linear augmented plane wave method implemented in Wien2 $\mathrm{K}^{58}$ in conjunction with Perdew-Burke-Ernzerhof generalized gradient approximation ${ }^{59}$ of the exchange correlation functional. DFT+DMFT is implemented on top of Wien2K and documented in ref. ${ }^{60}$. In the DFT+DMFT calculations, the electronic charge was computed self-consistently on DFT+DMFT density matrix. The quantum impurity problem was solved by the continuous time quantum Monte Carlo (CTQMC) method ${ }^{61,62}$ with a Hubbard $U=5.0 \mathrm{eV}$ and Hund's rule coupling $J=0.8 \mathrm{eV}$ in the paramagnetic state. ${ }^{6,63,64}$ Bethe-Salpeter equation is used to compute the dynamic spin susceptibility where the bare susceptibility is computed using the converged DFT+DMFT Green's function while the two-particle vertex is directly sampled using CTQMC method after achieving full self-consistency of DFT+DMFT density matrix. The detailed method of computing the dynamic spin susceptibility is documented in ref. ${ }^{6}$ and was shown to be able to compute accurately the spin dynamics of many iron pnictide superconductors. The experimental crystal structure (space group 14/mmm, \#139) of V-doped LiFeAs with lattice constants $a=$ $b=3.7914 \AA$ and $c=6.3639 \AA^{65}$ is used in the calculations. Virtual crystal approximation is employed to approximate the V-doping effect. In Fig. S7, we plot the calculated dynamic spin susceptibility from two inter-orbital scattering channels, which are $d_{x y}-d_{x z}$ and $d_{x y}-d_{y z}$ in LiFeAs and LiFe ${ }_{0.967^{-}}$ $\mathrm{V}_{0.033} \mathrm{As}$. The enhancement of electron scattering in both channels in $\mathrm{LiFe}_{0.967} \mathrm{~V}_{0.033} \mathrm{As}$ is closely associated with the Fermi surface nesting between the inner hole pockets $\left(d_{x z} / d_{y z}\right)$ and the inner electron pocket $\left(d_{x y}\right)$. Such an enhanced electron scattering between different orbitals is consistent with the "spin mixing" process as shown in Fig. $4 \mathrm{c}$ in the manuscript and drives the system into the $S=1 / 2$ state. Furthermore, such spin-state fluctuations might be responsible for the biquadratic exchange coupling, which is essential for the nematic instability existing extensively in iron pnictide/chalcogenide superconductors.

\section{DATA AVAILABILITY}

The neutron-scattering and numerical data used in this work, and in the figures of this manuscript, are available upon request from the corresponding authors.

\section{CODE AVAILABILITY}

The computer codes used to carry out the DFT+DMFT calculations used in this work are available upon request from the corresponding author.

Received: 23 June 2019; Accepted: 30 December 2019; Published online: 13 February 2020

\section{REFERENCES}

1. Lin, J. F. et al. Intermediate-spin ferrous iron in lowermost mantle post-perovskite and perovskite. Nat. Geosci. 1, 688-691 (2008) 
2. Bren, K. L., Eisenberg, R. \& Gray, H. B. Discovery of the magnetic behavior of hemoglobin: a beginning of bioinorganic chemistry. Proc. Natl Acad. Sci. USA 112, 13123-13127 (2015).

3. Stewart, G. R. Superconductivity in iron compounds. Rev. Mod. Phys. 83, 1589-1652 (2011).

4. Krüger, F., Kumar, S., Zaanen, J. \& van den Brink, J. Spin-orbital frustrations and anomalous metallic state in iron-pnictide superconductors. Phys. Rev. B 79, 054504 (2009).

5. Dai, P. C. Antiferromagnetic order and spin dynamics in iron-based superconductors. Rev. Mod. Phys. 87, 855 (2015).

6. Yin, Z. P., Haule, K. \& Kotliar, G. Kinetic frustration and the nature of the magnetic and paramagnetic states in iron pnictides and iron chalcogenides. Nat. Mater. 10, 932 (2011).

7. Mannella, N. The magnetic moment enigma in Fe-based high temperature superconductors. J. Phys. Condens. Matter 26, 473202 (2014).

8. Lorenzana, J., Seibold, G. \& Coldea, R. Sum rules and missing spectral weight in magnetic neutron scattering in the cuprates. Phys. Rev. B 72, 224511 (2005).

9. Wang, M. et al. Doping dependence of spin excitations and its correlations with high-temperature superconductivity in iron pnictides. Nat. Commun. 4, 2874 (2013).

10. Luo, H. Q. et al. Electron doping evolution of the anisotropic spin excitations in $\mathrm{BaFe}_{2-x} \mathrm{Ni}_{x} \mathrm{As}_{2}$. Phys. Rev. B 86, 024508 (2012).

11. Liu, M. S. et al. Nature of magnetic excitations in superconducting $B a \mathrm{Fe}_{1.9} \mathrm{Ni}_{0.1} \mathrm{As}_{2}$. Nat. Phys. 8, 376-381 (2012).

12. Luo, H. Q. et al. Electron doping evolution of the magnetic excitations in $\mathrm{BaFe}_{2}$ ${ }_{-} \mathrm{Ni}_{x} \mathrm{As}_{2}$. Phys. Rev. B 88, 144516 (2013).

13. Horigane, K. et al. Spin excitations in hole-overdoped iron-based superconductors. Sci. Rep. 6, 33303 (2016).

14. Carr, S. V. et al. Electron doping evolution of the magnetic excitations in $\mathrm{NaFe}_{1}$ ${ }_{-x} \mathrm{Co}_{x}$ As. Phys. Rev. B 93, 214506 (2016).

15. Qureshi, N. et al. Inelastic neutron-scattering measurements of incommensurate magnetic excitations on superconducting LiFeAs single crystals. Phys. Rev. Lett. 108, 117001 (2012).

16. Qureshi, N. et al. Fine structure of the incommensurate antiferromagnetic fluctuations in single-crystalline LiFeAs studied by inelastic neutron scattering. Phys. Rev. B 90, 144503 (2014).

17. Wang, M. et al. Effect of Li-deficiency impurities on the electron-overdoped LiFeAs superconductor. Phys. Rev. B 86, 144511 (2012).

18. Li, Y. et al. Orbital selective spin excitations and their impact on superconductivity of $\mathrm{LiFe}_{1-x} \mathrm{Co}_{x}$ As. Phys. Rev. Lett. 116, 247001 (2016).

19. Wang, Q. S. et al. Magnetic ground state of FeSe. Nat. Commun. 7, 12182 (2016).

20. Zaliznyak, I. A. et al. Unconventional temperature enhanced magnetism in $\mathrm{Fe}_{1,1}$ Te. Phys. Rev. Lett. 107, 216403 (2011).

21. Hirschfeld, P. J., Korshunov, M. M. \& Mazin, I. I. Gap symmetry and structure of Febased superconductors. Rep. Prog. Phys. 74, 124508 (2011).

22. de'Medici, L., Giovannetti, G. \& Capone, M. Selective Mott physics as a key to iron superconductors. Phys. Rev. Lett. 112, 177001 (2014).

23. Yu, R. \& Si, Q. Orbital-selective Mott phase in multiorbital models for iron pnictides and chalcogenides. Phys. Rev. B 96, 125110 (2017).

24. Komijani, Y. \& Kotliar, G. Analytical slave-spin mean-field approach to orbital selective Mott insulators. Phys. Rev. B 96, 125111 (2017).

25. Gastiasora, M. N. \& Anderson, B. M. Enhancement of magnetic stripe order in ironpnictide superconductors from the interaction between conduction electrons and magnetic impurities. Phys. Rev. Lett. 113, 067002 (2014).

26. Inosov, D. S. et al. Possible realization of an antiferromagnetic Griffiths phase in $\mathrm{Ba}\left(\mathrm{Fe}_{1-x} \mathrm{Mn}_{x}\right)_{2} \mathrm{As}_{2}$. Phys. Rev. B 87, 224425 (2013).

27. Tucker, G. S. et al. Competition between stripe and checkerboard magnetic instabilities in Mn-doped BaFe $\mathrm{As}_{2}$. Phys. Rev. B 86, 020503 (2012).

28. Song, Y. et al. A Mott insulator continuously connected to iron pnictide superconductors. Nat. Comm. 7, 13879 (2016).

29. Matt, C. E. et al. $\mathrm{NaFe}_{0.56} \mathrm{Cu}_{0.44} \mathrm{As}$ : a pnictide insulating phase induced by on-site Coulomb interaction. Phys. Rev. Lett. 117, 097001 (2016).

30. Xing, L. Y. et al. Observation of non-Fermi liquid behavior in hole-doped $\mathrm{LiFe}_{1}$ ${ }_{-x} V_{x}$ As. Phys. Rev. B 94, 094524 (2016).

31. Ong, T. T. \& Coleman, P. Local quantum criticality of an iron-pnictide tetrahedron. Phys. Rev. Lett. 108, 107201 (2012).

32. Zhang, J. H., Sknepnek, R. \& Schmalian, J. Spectral analysis for the iron-based superconductors: anisotropic spin fluctuations and fully gapped $s^{ \pm}$-wave superconductivity. Phys. Rev. B 82, 134527 (2010).

33. Chen, T. et al. Anisotropic spin fluctuations in detwinned FeSe. Nature Materials 18, 709 (2019).

34. Pfau, H. et al. Momentum dependence of the nematic order parameter in ironbased superconductors. Phys. Rev. Lett. 123, 066402 (2019).

35. Pfau, $\mathrm{H}$. et al. Detailed band structure of twinned and detwinned $\mathrm{BaFe}_{2} \mathrm{As}_{2}$ studied with angle-resolved photoemission spectroscopy. Phys. Rev. B 99, 035118 (2019).
36. Watson, M. D. et al. Probing the reconstructed Fermi surface of antiferromagnetic $\mathrm{BaFe}_{2} \mathrm{As}_{2}$ in one domain. npj Quant. Mater. 4, 36 (2019).

37. Tian, L. et al. Spin fluctuation anisotropy as a probe of orbital-selective holeelectron quasiparticle excitations in detwinned $\mathrm{Ba}\left(\mathrm{Fe}_{1-x} \mathrm{Co}_{x}\right) \mathrm{As}_{2}$. Phys. Rev. $\mathrm{B}$ 100, 134509 (2019).

38. Zhang, C. L. et al. Neutron scattering studies of spin excitations in hole-doped $\mathrm{Ba}_{0.67} \mathrm{~K}_{0.33} \mathrm{Fe}_{2} \mathrm{As}_{2}$ superconductor. Sci. Rep. 1, 115 (2011).

39. Harriger, L. W. et al. Nematic spin fluid in the tetragonal phase of $\mathrm{BaFe}_{2} \mathrm{As}_{2}$. Phys. Rev. B 84, 054544 (2011).

40. Lu, X. Y. et al. Spin waves in detwinned BaFe $\mathrm{As}_{2}$. Phys. Rev. Lett. 121, 067002 (2018).

41. Georges, A., de'Medici, L. \& Mravlje, J. Strong electronic correlations from Hund's coupling. Annu. Rev. Condens. Matter Phys. 4, 137-178 (2013).

42. Aron, C. \& Kotliar, G. Analytic theory of Hund's metals: a renormalization group perspective. Phys. Rev. B 91, 041110 (2015).

43. Stadler, K. M., Yin, Z. P., von Delft, J., Kotliar, G. \& Weichselbaum, A. Dynamical mean-field theory plus numerical renormalization-group study of spin-orbital separation in a three-band hund metal. Phys. Rev. Lett. 115, 136401 (2015).

44. Chaloupka, J. \& Khaliullin, G. Spin-state crossover model for the magnetism of iron pnictides. Phys. Rev. Lett. 110, 207205 (2013).

45. Gütlich, P. \& Goodwin, H. A. (eds) Spin Crossover in Transition Metal Compounds I (Springer, Berlin, 2004).

46. Gretarsson, H. et al. Spin-state transition in the Fe pnictides. Phys. Rev. Lett. 110, 047003 (2013)

47. Klingeler, R. et al. Local antiferromagnetic correlations in the iron pnictide superconductors $\mathrm{LaFeAsO}_{1-x} \mathrm{~F}_{x}$ and $\mathrm{Ca}\left(\mathrm{Fe}_{1-x} \mathrm{Co}_{x}\right)_{2} \mathrm{As}_{2}$ as seen via normal-state susceptibility. Phys. Rev. B 81, 024506 (2010).

48. Yang, R. et al. Observation of an emergent coherent state in the iron-based superconductor $\mathrm{KFe}_{2} \mathrm{As}_{2}$. Phys. Rev. B 96, 201108 (2017).

49. Wiecki, P. et al. Pressure dependence of coherence-incoherence crossover behavior in $\mathrm{KFe}_{2} \mathrm{As}_{2}$ observed by resistivity and ${ }^{75} \mathrm{As}-\mathrm{NMR} / \mathrm{NQR}$. Phys. Rev. B 97, 064509 (2018).

50. Hardy, F. et al. Evidence of strong correlations and coherence-incoherence crossover in the iron pnictide superconductor $\mathrm{KFe}_{2} \mathrm{As}_{2}$. Phys. Rev. Lett. 111, 027002 (2013).

51. Miao, H. et al. Orbital-differentiated coherence-incoherence crossover identified by photoemission spectroscopy in LiFeAs. Phys. Rev. B 94, 201109 (2016).

52. Lee, P. A., Nagaosa, N. \& Wen, X. G. Doping a Mott insulator: physics of hightemperature superconductivity. Rev. Mod. Phys. 78, 17 (2006).

53. Wang, W. Y. et al. Orbital selective neutron spin resonance in underdoped superconducting $\mathrm{NaFe}_{0.985} \mathrm{As}_{0.015}$. Phys. Rev. B 95, 094519 (2017).

54. Wang, M. et al. Antiferromagnetic spin excitations in single crystals of nonsuperconducting $\mathrm{Li}_{1-x} \mathrm{FeAs}$. Phys. Rev. B 83, 220515 (2011).

55. Xing, L. Y. et al. The anomaly $\mathrm{Cu}$ doping effects on LiFeAs superconductors. J. Phys. Condens. Matter 26, 435703 (2014).

56. Kajimoto, R. et al. The Fermi chopper spectrometer 4SEASONS at J-PARC. J. Phys. Soc. Jpn. 80, SB025 (2011).

57. Kotliar, G. et al. Electronic structure calculations with dynamical mean-field theory. Rev. Mod. Phys. 78, 865 (2006).

58. Blaha, P., Schwarz, K., Madsen, G., Kvasnicka, D. \& Luitz, J. WIEN2K, An Augmented Plane Wave+Local Orbitals Program for Calculating Crystal Properties (Karlheinz Schwarz, Techn. Universität Wien, Wien, 2001).

59. Perdew, J. P., Burke, K. \& Ernzerhof, M. Generalized gradient approximation made simple. Phys. Rev. Lett. 77, 3865 (1996).

60. Haule, K., Yee, C.-H. \& Kim, K. Dynamical mean-field theory within the fullpotential methods: electronic structure of Celrln ${ }_{4}, C$ Coln ${ }_{5}$, and CeRhln ${ }_{5}$. Phys. Rev. B 81, 195107 (2010).

61. Haule, K. Quantum Monte Carlo impurity solver for cluster dynamical mean-field theory and electronic structure calculations with adjustable cluster base. Phys. Rev. B 75, 155113 (2007)

62. Werner, P., Comanac, A., de'Medici, L., Troyer, M. \& Millis, A. J. Continuous-time solver for quantum impurity models. Phys. Rev. Lett. 97, 076405 (2006).

63. Yin, Z. P., Haule, K. \& Kotliar, G. Magnetism and charge dynamics in iron pnictides. Nat. Phys. 7, 294 (2011).

64. Yin, Z. P., Haule, K. \& Kotliar, G. Spin dynamics and orbital-antiphase pairing symmetry in iron-based superconductors. Nat. Phys. 10, 845 (2014).

65. Tapp, J. H. et al. LiFeAs: an intrinsic FeAs-based superconductor with $T_{\mathrm{c}}=18 \mathrm{~K}$. Phys. Rev. B 78, 060505(R) (2008).

\section{ACKNOWLEDGEMENTS}

The neutron-scattering work at Beijing Normal University is supported by the Fundamental Research Funds for the Central Universities (Grant Nos. 310432101 and 2014JJCB27) and the National Natural Science Foundation of China (Grant No. 
11734002) (to X.L.). Z.Y. was supported by the NSFC (Grant No. 11674030), the Fundamental Research Funds for the Central Universities (Grant No. 310421113), and the National Key Research and Development Program of China grant 2016YFA0302300. The calculations used high-performance computing clusters at BNU in Zhuhai and the National Supercomputer Center in Guangzhou. The neutronscattering work at Rice is supported by the U.S. DOE BES under contract no. DESC0012311 (to P.D.). The neutron experiment at the Materials and Life Science Experimental Facility of the J-PARC was performed under a user program 2017 B0216.

\section{AUTHOR CONTRIBUTIONS}

P.D., G.T., X.L., and C.J. designed the research. Single crystals were grown by G.D. and L.X. under the instructions of X.W. and C.J. Neutron-scattering experiments were carried out by Z.X., G.T., Y.R., L.T., Y.L., P.L., H.W., Y.W., R.K., K.I., and D.L.A. Theoretical calculations were carried out by Z.Y. The paper was written by P.D., Y.L., Z.X., and Z.Y. All authors made comments on the paper.

\section{COMPETING INTERESTS}

The authors declare no competing interests.

\section{ADDITIONAL INFORMATION}

Supplementary information is available for this paper at https://doi.org/10.1038/ s41535-020-0212-x.
Correspondence and requests for materials should be addressed to Z.Y., G.T. or P.D.

Reprints and permission information is available at http://www.nature.com/ reprints

Publisher's note Springer Nature remains neutral with regard to jurisdictional claims in published maps and institutional affiliations.

Open Access This article is licensed under a Creative Commons Attribution 4.0 International License, which permits use, sharing, adaptation, distribution and reproduction in any medium or format, as long as you give appropriate credit to the original author(s) and the source, provide a link to the Creative Commons license, and indicate if changes were made. The images or other third party material in this article are included in the article's Creative Commons license, unless indicated otherwise in a credit line to the material. If material is not included in the article's Creative Commons license and your intended use is not permitted by statutory regulation or exceeds the permitted use, you will need to obtain permission directly from the copyright holder. To view a copy of this license, visit http://creativecommons. org/licenses/by/4.0/.

(c) The Author(s) 2020 